\title{
The Design of Robotic Arm Adaptive Fuzzy Controller Based on Oscillator and Differentiator
}

\author{
https://doi.org/10.3991/ij ijoe.v15i05.8895 \\ Min Wan, Qinglan Tian ${ }^{(凶)}$,Chuanhong Sun, Xiuyuan Yi \\ Southwest Petroleum University, Chengdu, China \\ 451755342 @qq. com
}

\begin{abstract}
This paper mainly obtains the value of the state variable by designing the differentiator. The value of the state variable is necessary to track the manipulator with adaptive fuzzy controller. But difficult to obtain directly. In this paper, a model based on the dynamics analysis of robotic arm was build to design the second order oscillator and the Second Order differentiator in finite time to obtain the value of each state variable. The designed adaptive fuzzy controller for robotic arm achieved high accuracy in trace tracking. Simulation results of two-link robotic arm show the adaptive fuzzy controller for robotic arm based on differentiators is adaptable, flexible. This controller is simple to design, easy to implement, and has a good value for the application of robotic arm system.
\end{abstract}

Keywords-Robotic arm, Adaptive Fuzzy Controller, Second Order Oscillator, Second Order differentiator in finite time

\section{$1 \quad$ Introduction}

Robotic arm has been developing continuously; it can do a lot of repeated, monotonous long-hour works or high-risk jobs in harsh environments or instead of mankind [1]. In order to make the robotic arm accomplish the movement in the specified path, its trace must be tracked [2]. Robotic arm system is a complex multiple-input-multiple-output nonlinear system with significant coupling, and there also exist load changes, stochastic disturbances, measurement error and many other uncertainties. Thus, it is difficult to build an accurate mathematical model of the robotic arm. Fuzzy system does not need to rely on accurate mathematical model, in paper [3] [4] adaptive fuzzy controller was used in tracking the robotic arm and achieved good results. However, when adopting the fuzzy adaptive methods the condition of the system state is assumed that it can be measured directly, [5] is based on the assumption that the system state can be measured directly. In [6] - [10] state observer is used in estimating the state variables; however, it is more than complex to design the state observer. In this article, while the state condition is immeasurable, the second order oscillator and the second order differentiator that converges in finite time are introduced to obtain the 
value of state variables. In this thesis, the adaptive fuzzy controller realized high-precision tracking of robotic arm.

\section{Dynamic Modeling and Analysis of Robotic Arm}

According to Lagrange equation $L=K-P$, where $L$ is the Lagrange function; $K$ is the total kinetic energy of the robotic arm; $P$ is the potential energy of it. Taking into account the friction and other disturbance vectors, we have the general dynamic equation of a double-joint robotic arm system, which reflects the functional relation among motion displacement, velocity, acceleration [11]:

$$
\tau=D(q) \ddot{q}+V(q, \dot{q}) \dot{q}+\mathrm{H}(q)+\mathrm{J}(q, \dot{q})+d(q, \dot{q})
$$

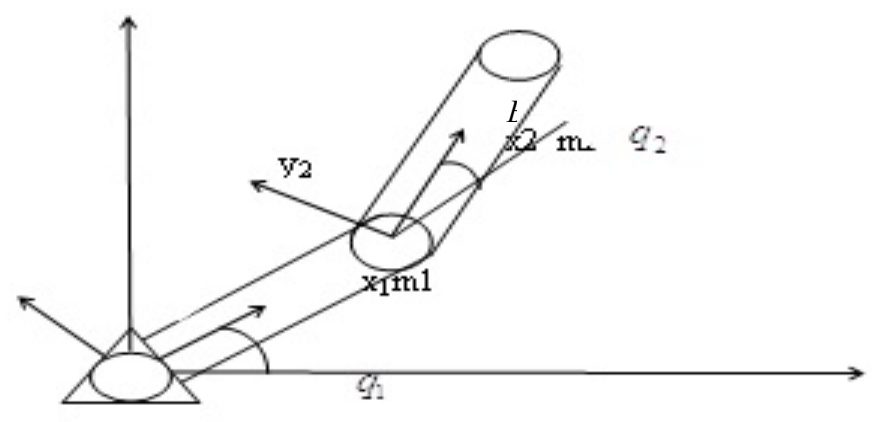

Fig. 1. Double-hinged robotic arm

In this equation $q=$ generalized displacement; $\dot{q}=$ generalized speed; $\ddot{q}=$ generalized acceleration; $\tau=$ driving torque vector; $D(q) \in R^{2 \times 2}$ is the inertia matrix of the robotic arm; $V(q, \dot{q}) \in R^{2 \times 2}$ represents the centrifugal and Coriolis matrix; $\mathrm{H}(q) \in R^{2 \times 1}$ is the gravity matrix; $\mathrm{J}(q, \dot{q}) \in R^{2 \times 1}$ is the friction matrix; $d(q, \dot{q}) \in R^{2 \times 1}$ is the additional disturbance vectors.

The robot arm system is a complex nonlinear system with uncertain factors which is difficult to establish accurate mathematical models. Therefore, an adaptive fuzzy controller independent of precise mathematical model is designed in this paper. 


\section{Design of Adaptive Fuzzy Controller}

Consider MIMO Nonlinear Systems [12]:

$$
\begin{gathered}
y_{1}^{\left(r_{1}\right)}=f_{1}(x)+\sum_{j=1}^{p} g_{1 j}(x) u_{j} \\
\vdots \\
y_{p}^{\left(r_{p}\right)}=f_{p}(x)+\sum_{j=1}^{p} g_{p j}(x) u_{j}
\end{gathered}
$$

where the state variables $x=\left[y_{1}, \dot{y}_{1}, \cdots, y_{1}^{\left(r_{1}-1\right)}, \cdots, y_{p}, y_{p}^{\left(r_{p}-1\right)}\right]^{T}$ is measurable, $u=\left[u_{1}, \cdots, u_{p}\right]^{T}$ is input variables; $y=\left[y_{1}, \cdots, y_{p}\right]^{T}$ is output variables; $f_{i}(x)$ and $g_{i j}(x)$ are unknown smooth nonlinear functions $(i, j=1,2, \cdots, p)$.

In this equation

$$
\begin{gathered}
y^{(r)}=\left[y_{1}^{r_{1}}, \cdots y_{p}^{r_{p}}\right]^{T} \\
F(x)=\left[f_{1}(x), \cdots f_{p}(x)\right]^{T} \\
G(x)=\left[\begin{array}{ccc}
g_{11}(x) & \cdots & g_{1 p}(x) \\
\vdots & \ddots & \vdots \\
g_{p 1}(x) & \cdots & g_{p p}(x)
\end{array}\right]
\end{gathered}
$$

Then formula (2) can be expressed as

$$
y^{(r)}=F(x)+G(x) u
$$

By designing the control law $u(t)$, all variables are ensured to be bounded, and the output of the system can track the desired trajectory $y_{d}(t)=\left[y_{d 1}(t), \cdots, y_{d p}(t)\right]^{T}$. 
Hypothesis 1: $G(x)$ It is a positive definite matrix, and there exist a real number $\sigma_{0}>0$, which satisfies $G(x) \geq \sigma_{0} I_{p}$

Hypothesis 2: Desired trajectory $y_{d i}(t), i=1,2, \cdots, p$ is bounded, and there is $r_{i}$ order derivative, and each order of the derivative are also bounded.

Hypothesis 1 can guarantee that the inverse of $G(x)$ exists, then Formula (2) can be expressed as a linear form of the static state feedback, although assumptions are strictly limited to a MIMO system, the robotic arm system can satisfy the following assumptions:

Define trajectory tracking error as

$$
\begin{gathered}
e_{1}(t)=y_{d 1}(t)-y_{1}(t) \\
\vdots \\
e_{p}(t)=y_{d p}(t)-y_{p}(t)
\end{gathered}
$$

Define the error function of filter tracking as:

$$
\begin{gathered}
s_{1}(t)=\left(\frac{d}{d t}+\lambda_{1}\right)^{r_{1}-1} e_{1}(t), \lambda_{1}>0 \\
s_{p}(t)=\left(\frac{d}{d t}+\lambda_{p}\right)^{r_{p}-1} e_{p}(t), \lambda_{p}>0
\end{gathered}
$$

As Formula (5) shows, when $s_{i} \rightarrow 0, e_{i} \rightarrow 0(i=1,2, \cdots, p)$, the control object can be transformed into the $s_{i} \rightarrow 0(i=1,2, \cdots, p)$.

According to Newton's binomial theorem

$$
(a+b)^{n}=\sum_{i=0}^{n} C_{n}^{i} a^{i} b^{n-i}
$$


Where $C_{n}^{i}=\frac{n !}{(n-i) ! i !}$ is binomial expansion coefficient.

Apply Formula (6) to Formula (5) get

$$
\begin{aligned}
s_{i}(t) & =\left(\frac{d}{d t}+\lambda_{i}\right)^{r_{i}-1} e_{i}(t)=\sum_{j=0}^{r_{i}-1} \frac{\left(r_{i}-1\right) !}{\left(r_{i}-1-j\right) ! j !}\left(\frac{d}{d t}\right)^{j} e_{i}(t) \lambda_{i}^{r_{1}-1-j} \\
& =\sum_{j=1}^{r_{i}} \frac{\left(r_{i}-1\right) !}{\left(r_{i}-j\right) !(j-1) !}\left(\frac{d}{d t}\right)^{j-1} e_{i}(t) \lambda_{i}^{r_{1}-j}
\end{aligned}
$$

then

$$
\begin{aligned}
& \dot{s}_{i}(t)=\sum_{j=1}^{r_{i}} \frac{\left(r_{i}-1\right) !}{\left(r_{i}-j\right) !(j-1) !} e_{i}^{(j)} \lambda_{i}^{r_{i}-j}=e_{i}^{\left(r_{i}\right)}+\sum_{j=1}^{r_{i}-1} \frac{\left(r_{i}-1\right) !}{\left(r_{i}-j\right) !(j-1) !} e^{(j)}(t) \lambda_{i}^{r_{i}-j} \\
& =y_{d_{i}}^{\left(r_{i}\right)}-y_{i}^{\left(r_{i}\right)}+\sum_{j=1}^{r_{i}-1} \frac{\left(r_{i}-1\right) !}{\left(r_{i}-j\right) !(j-1) !} e_{i}^{(j)}(t) \lambda_{i}^{r_{i}-j} \\
& =y_{d_{i}}^{\left(r_{i}\right)}-f_{i}(x)-\sum_{j=1}^{i} g_{i j}(x) u_{j}+\sum_{j=1}^{r_{i}-1} \frac{\left(r_{i}-1\right) !}{\left(r_{i}-j\right) !(j-1) !} e_{i}^{(j)}(t) \lambda_{i}^{r_{i}-j}
\end{aligned}
$$

which is

$$
\begin{gathered}
\dot{s}_{1}(t)=v_{1}-f_{1}(x)-\sum_{j=1}^{p} g_{1 j}(x) u_{j} \\
\vdots \\
\dot{s}_{p}(t)=v_{p}-f_{p}(x)-\sum_{j=1}^{p} g_{p j}(x) u_{j}
\end{gathered}
$$

where

$$
\begin{gathered}
v_{1}=y_{d_{1}}^{\left(r_{1}\right)}+\beta_{1, r_{1}-1} e_{1}^{\left(r_{1}-1\right)}+\beta_{1,1} \dot{e}_{1} \\
\vdots \\
v_{p}=y_{d_{p}}^{\left(r_{p}\right)}+\beta_{p, r_{p}-1} e_{p}^{\left(r_{p}-1\right)}+\beta_{p, p} \dot{e}_{p} \\
\beta_{i, j}=\frac{\left(r_{i}-1\right) !}{\left(r_{i}-j\right) !(j-1) !} \lambda_{i}^{r_{i}-j}, i=1, \cdots, p, j=1, \cdots, r_{i}-1
\end{gathered}
$$




$$
\begin{aligned}
& \quad \text { Make } s(t)=\left[s_{1}(t), \cdots, s_{p}(t)\right]^{T}, v(t)=\left[v_{1}(t), \cdots, v_{p}(t)\right]^{T} \text { then } \\
& \dot{s}=v-F(x)-G(x) u
\end{aligned}
$$

In a practical system, the nonlinear function $f_{i}(x)$ and $g_{i j}(x)$ are unknown, fuzzy system can be used to approximate the non-linear function.

Assuming the fuzzy system is a mapping from $U \subseteq R^{n}$ to $R$, and $U=U_{1} \times \cdots \times U_{n}, U_{i} \subset R(i=1, \cdots, n)$. The lth fuzzy rules can be expressed as

$$
R^{(l)}: \mathrm{IF}^{x_{1}}{ }_{\text {is }} F_{1}^{l} \text { and } \cdots \text { and } x_{n} \text { is } F_{n}^{l} \text {, THEN } y^{l}{ }_{\text {is }} C^{l}(l=1,2, \cdots, M)
$$

where $x=\left[x_{1}, x_{2}, \cdots, x_{n}\right]^{T} \in U, y \in V \subset R$ are input and output of the fuzzy system.

Introduce Singleton fuzzifier, product inference engine and the center average defuzzifier into the design of fuzzy systems, the output of the fuzzy system

$$
y(x)=\frac{\sum_{l=1}^{M}-l\left(\prod_{i=1}^{n} \mu_{F_{i}}^{l}\left(x_{i}\right)\right)}{\sum_{l=1}^{M}\left(\prod_{i=1}^{n} \mu_{F_{i}}^{l}\left(x_{i}\right)\right)}
$$

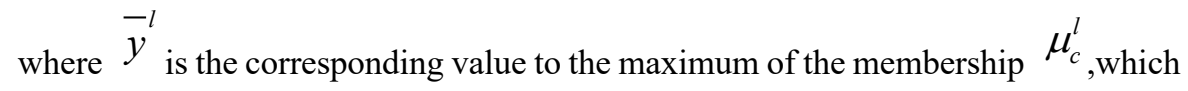
is $\mu_{c}^{l}\left(\bar{y}^{l}\right)=1$

then Formula (12) can be written as then Formula (12) can be written as

$$
y(x)=\xi^{T}(x) \theta
$$


where $\theta=\left[y^{1}, \cdots, y^{M}\right]$ is the parameter vector, $\xi(x)=\left[\xi_{1}(x), \cdots, \xi_{M}(x)\right]^{T}$ is the regression vector. Define

$$
\xi_{l}(x)=\frac{\prod_{i=1}^{n} \mu_{F_{i}}^{l}\left(x_{i}\right)}{\sum_{l=1}^{M}\left(\prod_{i=1}^{n} \mu_{F_{i}}^{l}\left(x_{i}\right)\right)}
$$

And the fuzzy system for approximating the nonlinear functions $f_{i}(x)$ and $g_{i j}(x)$ is

$$
\begin{gathered}
\hat{f}_{i}\left(x, \theta_{f_{i}}\right)=\xi_{f_{i}}^{T}(x) \theta_{f_{i}}, i=1, \cdots, p \\
\hat{g}_{i j}\left(x, \theta_{g_{i j}}\right)=\xi_{g_{i j}}^{T}(x) \theta_{g_{i j}}, i=1, \cdots, p
\end{gathered}
$$

where $\xi_{f_{i}}(x)$ and $\xi_{g_{i j}}(x)$ is fuzzy basis function vectors; $\theta_{f_{i}}$ and $\theta_{g_{i j}}$ is the adaptive parameter vector.

Assume the best-fit parameters of $\theta_{f_{i}}$ and $\theta_{g_{i j}}$ is $\theta_{f_{i}}^{*}$ and $\theta_{g_{i j}}^{*}$, the minimum fuzzy approximation errors are $\varepsilon_{f_{i}}(x)$ and $\varepsilon_{g_{i j}}(x)$, which are defined as follows:

$$
\begin{gathered}
\theta_{f_{i}}^{*}=\arg \min _{\theta_{f_{i}}}\left\{\sup _{x \in D_{x}}\left|f_{i}(x)-\hat{f}_{i}\left(x, \theta_{f_{i}}\right)\right|\right\} \\
\theta_{g_{i j}}^{*}=\arg \min _{\theta_{g_{i j}}}\left\{\sup _{x \in D_{x}}\left|g_{i j}(x)-\hat{g}_{i j}\left(x, \theta_{g_{i j}}\right)\right|\right\} \\
\widetilde{\theta}_{f_{i}}=\theta_{f_{i}}^{*}-\theta_{f_{i}}, \widetilde{\theta}_{g_{i j}}=\boldsymbol{\theta}_{g_{i j}}^{*}-\boldsymbol{\theta}_{g_{i j}} \\
\varepsilon_{f_{i}}(x)=f_{i}(x)-\hat{f}_{i}\left(x, \theta_{f_{i}}^{*}\right) \\
\varepsilon_{g_{i j}}(x)=g_{i j}(x)-\hat{g}_{i j}\left(x, \theta_{g_{i j}}^{*}\right)
\end{gathered}
$$


Assuming compact set $D_{x}$ is large enough, for all $x \in D_{x}$, the minimum approximation error is bounded, i.e. $\left|\varepsilon_{f_{i}}(x)\right| \leq \bar{\varepsilon}_{f_{i}}(x),\left|\varepsilon_{g_{i j}}(x)\right| \leq \bar{\varepsilon}_{g_{i j}}(x)$,among them $\bar{\varepsilon}_{f_{i}}, \bar{\varepsilon}_{g_{i j}}(x)$. It is a known constant.

Set

$$
\begin{aligned}
& \hat{F}(x)=\left[\hat{f}_{1}(x), \cdots, \hat{f}_{p}(x)\right]^{T} \\
& \hat{G}(x)=\left[\begin{array}{ccc}
\hat{g}_{11}(x) & \cdots & \hat{g}_{1 p}(x) \\
\vdots & \ddots & \vdots \\
\hat{g}_{p 1}(x) & \cdots & \hat{g}_{p p}(x)
\end{array}\right] \\
& \varepsilon_{f}(x)=\left[\varepsilon_{f_{1}}(x), \cdots, \varepsilon_{f_{p}}(x)\right]^{T} \\
& \varepsilon_{g}(x)=\left[\begin{array}{ccc}
\varepsilon_{g_{11}}(x) & \cdots & \varepsilon_{g_{1 p}}(x) \\
\vdots & \ddots & \vdots \\
\varepsilon_{g_{p 1}}(x) & \cdots & \varepsilon_{g_{p p}}(x)
\end{array}\right] \\
& \bar{\varepsilon}_{f}(x)=\left[\bar{\varepsilon}_{f_{1}}(x), \cdots, \bar{\varepsilon}_{f_{p}}(x)\right]^{T} \\
& \bar{\varepsilon}_{g}(x)=\left[\begin{array}{ccc}
\bar{\varepsilon}_{g_{11}}(x) & \cdots & \bar{\varepsilon}_{g_{1 p}}(x) \\
\vdots & \ddots & \vdots \\
\bar{\varepsilon}_{g_{p 1}}(x) & \cdots & \bar{\varepsilon}_{g_{p p}}(x)
\end{array}\right]
\end{aligned}
$$

then

$$
\begin{aligned}
& F(x)-\hat{F}\left(x, \theta_{f}\right)=\hat{F}\left(x, \theta_{f}^{*}\right)-\hat{F}\left(x, \theta_{f}\right)+\varepsilon_{f}(x) \\
& G(x)-\hat{G}\left(x, \theta_{g}\right)=\hat{G}\left(x, \theta_{g}^{*}\right)-\hat{G}\left(x, \theta_{g}\right)+\varepsilon_{g}(x)
\end{aligned}
$$


Use $\hat{F}\left(x, \theta_{f}\right)$ and $\hat{G}\left(x, \theta_{g}\right)$ to replace $F(x)$ and $G(x)$, the control law is

$$
u_{c}=\hat{G}^{-1}\left(x, \theta_{g}\right)\left(-\hat{F}\left(x, \theta_{f}\right)+v+K_{0} s\right)
$$

Because $\hat{G}\left(x, \theta_{g}\right)$ is given by online estimating the value of $\theta_{g}$, it is difficult to ensure that $\hat{G}\left(x, \theta_{g}\right)$ is non-singularity. Thus, introducing the generalized inverse $\hat{G}^{T}\left(x, \theta_{g}\right)\left[\varepsilon_{0} I_{p}+\hat{G}\left(x, \theta_{g}\right) \hat{G}^{T}\left(x, \theta_{g}\right)\right]^{-1}$ to replace $\hat{G}^{-1}\left(x, \theta_{g}\right)$, then control law is

$$
u_{r}=\frac{s\left|s^{T}\right|\left(\bar{\varepsilon}_{f}+\bar{\varepsilon}_{g}\left|u_{c}\right|+\left|u_{0}\right|\right)}{\sigma_{0}\|s\|^{2}+\delta}
$$

where $\varepsilon_{0}$ is a positive real number arbitrarily small, $I_{p}$ is the unit matrix.

In order to reduce the modeling errors, the robust controls $u_{r}$ was introduced, the control law is

$$
\begin{gathered}
u=u_{c}+u_{r} \\
u_{r}=\frac{s\left|s^{T}\right|\left(\bar{\varepsilon}_{f}+\bar{\varepsilon}_{g}\left|u_{c}\right|+\left|u_{0}\right|\right)}{\sigma_{0}\|s\|^{2}+\delta} \\
u_{0}=\varepsilon_{0}\left[\varepsilon_{0} I_{p}+\hat{G}\left(x, \theta_{g}\right) \hat{G}^{T}\left(x, \theta_{g}\right)\right]^{-1}\left(-\hat{F}\left(x, \theta_{f}\right)+v+K_{0} s\right) \\
\text { where } \partial \text { It is a time-varying parameter. }
\end{gathered}
$$

Adaptive control law is

$$
\begin{gathered}
\dot{\theta}_{f_{i}}=-\eta_{f_{i}} \xi_{f_{i}}(x) s_{i} \\
\dot{\theta}_{g_{i j}}=-\eta_{g_{i j}} \xi_{g_{i j}}(x) s_{i} u_{c j}
\end{gathered}
$$




$$
\dot{\delta}=-\eta_{0} \frac{\left|s^{T}\right|\left(\bar{\varepsilon}_{f}+\bar{\varepsilon}_{g}\left|u_{c}\right|+\left|u_{0}\right|\right)}{\sigma_{0}\|s\|^{2}+\delta}
$$

where $\eta_{f_{i}}>0, \eta_{g_{i j}}>0, \eta_{0}>0, \delta(0)>0$.

While design the fuzzy controller, we assume that the state variables $x=\left[y_{1}, \dot{y}_{1}, \cdots, y_{1}^{\left(r_{1}-1\right)}, \cdots, y_{p}, y_{p}^{\left(r_{p}-1\right)}\right]^{T}$ is measurable. For some immeasurable state, the following differentiator is adopted.

\section{Design of Differentiator}

\subsection{Second-order Oscillation Link}

$V(s)$ and $X(s)$ are the Laplace transform of input $v(t)$ and output $x(t)$, the function of the second-order oscillation link is

$$
\frac{X(s)}{V(s)}=\frac{\omega_{n}^{2}}{s^{2}+2 \xi \omega_{n} s+\omega_{n}^{2}}
$$

set $^{x_{1}}(t)=x(t), x_{2}(t)=\dot{x}(t)$,then by the formula (32) available:

$$
\left\{\begin{array}{l}
\dot{x}_{1}=x_{2} \\
\dot{x}_{2}=-\omega_{n}^{2} x_{1}+\omega_{n}^{2} v(t)-2 \xi \omega_{n} x_{2}
\end{array}\right.
$$

The Laplace transformation of Formula (33) is

$$
\left\{\begin{array}{l}
s X_{1}(s)=X_{2}(s) \\
s X_{2}(s)=-\omega_{n}^{2} X_{1}(s)+\omega_{n}^{2} V(s)-2 \xi \omega_{n} X_{2}(s)
\end{array}\right.
$$

so 


$$
\frac{X_{2}(s)}{V(s)}=\frac{s}{\left(\frac{1}{\omega_{n}^{2}} s^{2}+\frac{2 \zeta}{\omega_{n}} s+1\right)}
$$

Available

$$
\lim _{\omega_{n} \rightarrow \infty} \frac{X_{2}(s)}{V(s)}=s
$$

Therefore, when ${ }^{\omega_{n}}$ large enough, the system can eliminate the high frequency noise, and the system output $x_{2}(t)$, i.e. derivative $\dot{v}^{\dot{v}(t)}$ of the tracking signal $v(t)$.

\subsection{Second Order differentiator in finite time}

If using the second-order oscillation link to obtain the derivative of signal, theoretically it only convergence when time goes to infinity, so the Second Order differentiator in finite time was adopted. The Second Order differentiator in finite time can quickly converge in finite time, and there is no chattering occurs. In addition to the derivative of the signal that is derivable, the differentiator can also obtain some non-smooth generalized derivatives.

Hereby introducing four assumptions:

Hypothesis 1: Assuming the equilibrium of the following system is a stable in finite time

$$
\left\{\begin{array}{l}
\dot{\tilde{z}}_{1}=\tilde{z}_{2} \\
\quad \vdots \\
\dot{\tilde{z}}_{n-1}=\tilde{z}_{n} \\
\dot{\tilde{z}}_{n}=f\left(\tilde{z}_{1}, \tilde{z}_{2}, \cdots, \tilde{z}_{n}\right)
\end{array}\right.
$$


And the stop time function $T_{f}$ is continuous at the zero point, where $f()$ is continuous and $f(0)=0$.N is the open neighborhood of the zero point. Then for the system formula (40), there is a continuous function $V$ that met: 1) $V$ is positive defi-

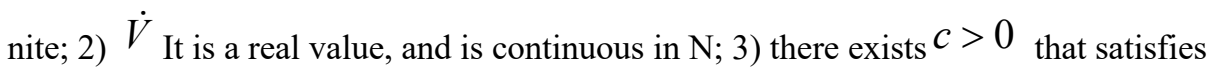

$$
\dot{V}+c V \leq 0
$$

Hypothesis 2: There is a Lipschitz Function that satisfies Lyapunov Function, function $V$ satisfies the Formula (38) and the Lipschitz Constant of Lyapunov Function is $\mathrm{M}$.

Hypothesis 3: For a system formula (37), there are $\rho_{i} \in(0,1], i=0,1, \cdots, n-1$ and a non-negative constant $\bar{a}_{\text {satisfy }}$

$$
\left|f\left(\tilde{z}_{1}, \tilde{z}_{2}, \cdots, \tilde{z}_{n}\right)-f\left(\bar{z}_{1}, \bar{z}_{2}, \cdots, \bar{z}_{n}\right)\right| \leq \bar{a} \sum_{i=1}^{n}\left|\tilde{z}_{i}-\bar{z}_{i}\right|^{\rho_{i-1}}
$$

Hypothesis 4: Suppose $\mathrm{e}^{v(t)}$ is a segment signal that is continuously differentiable, and satisfies the following properties: throughout the whole time domain, $n-2$ order derivative of signal $v(t)$ exists, and at some point $t_{j}(j=1, \cdots, k)$, the $n-1$ order is non-conductive. The $n-1$ order left derivative $v_{-}^{(n-1)}\left(t_{j}\right)$ and right derivative $v_{+}^{(n-1)}\left(t_{j}\right)$ of signal $v(t)$ both exist, and $v_{-}^{(n-1)}\left(t_{j}\right) \neq v_{+}^{(n-1)}\left(t_{j}\right),(j=1, \cdots, k)$.

Based on the above assumptions, the second-order differentiator that converges in finite time is designed as follows: 


$$
\left\{\begin{array}{l}
\dot{x}_{1}=x_{2} \\
\varepsilon^{2} \dot{x}_{2}=u \\
y=x_{2}
\end{array}\right.
$$

$$
u=-\operatorname{sat}_{\varepsilon_{b}}\left\{\operatorname{sgn}\left(\varphi_{a}\left(x_{1}-v(t), \varepsilon x_{2}\right)\right)\left|\varphi_{a}\left(x_{1}-v(t), \varepsilon x_{2}\right)\right|^{\frac{\alpha}{2-\alpha}}\right\}-\operatorname{sat}_{\varepsilon_{b}}\left\{\operatorname{sgn}\left(x_{2}\right)\left|\varepsilon x_{2}\right|^{\alpha}\right\}
$$

where

$$
\begin{gathered}
\left.\varphi_{a}\left(x_{1}-v(t), \varepsilon x_{2}\right)\left|=x_{1}-v(t)+\frac{1}{2-\alpha} \operatorname{sgn}\left(x_{2}\right)\right| \varepsilon x_{2}\right|^{2-\alpha} \\
\operatorname{sat}_{\varepsilon_{b}}(x)= \begin{cases}x & |x|<\varepsilon_{b} \\
\varepsilon_{b} \operatorname{sgn}(x) & |x| \geq \varepsilon_{b}\end{cases}
\end{gathered}
$$

There exist $\gamma>0$ (where, $\rho \lambda>2$ and $\rho=\min \{\alpha, \alpha /(2-\alpha)\}=$ $\alpha /(2-\alpha))$, for $t \geq \varepsilon \Gamma(\Xi(\varepsilon) e(0)), i=1$, where $\Xi(\varepsilon)=\operatorname{diag}\left\{1, \varepsilon, \cdots, \varepsilon^{n-1}\right\}$, $t_{j}>t \geq t_{j-1}+\varepsilon \Gamma\left(\Xi(\varepsilon) e\left(t_{j-1}\right)\right), j=1, \cdots, k+1, i=2$, thus we have

$$
x_{i}-v^{(i-1)}(t)=\mathrm{O}\left(\varepsilon^{\rho \lambda-i+1}\right)
$$

and $x_{2}\left(t_{j}\right)-v_{-}^{\prime}(t)=\mathrm{O}\left(\varepsilon^{\rho \lambda-1}\right), j=1, \cdots, k$ established, where $\varepsilon$ is the perturbation parameter, $\mathrm{O}\left(\varepsilon^{\rho \lambda-i+1}\right)$ represents that the approximation error between $x_{i}$ and $v^{i-1}(t)$ is $\varepsilon^{\rho \lambda-i+1}$ order, when $\alpha \in(0,1)$. In fact, the system

$$
\left\{\begin{array}{l}
\dot{x}_{1}=x_{2} \\
\dot{x}_{2}=-s a t_{\varepsilon_{b}}\left\{\operatorname{sgn}\left(\varphi_{a}\left(x_{1}, x_{2}\right)\right) \mid \varphi_{a}\left(x_{1}, x_{2}\right) \frac{\alpha}{\left.\right|^{2-\alpha}}\right\}-s a t_{\varepsilon_{b}}\left\{\operatorname{sgn}\left(x_{2}\right)\left|x_{2}\right|^{\alpha}\right\}
\end{array}\right.
$$


Converges at original point in a limited time $T_{f}$, and satisfies assumptions $1 \sim 4$. For Formula (47), when $\tau_{1}<\tau_{2}\left(i=1,2, \forall \tau_{1}, \tau_{2} \in[0, \infty)\right) \quad$, we have $\left|x_{i}\left(\tau_{2}\right)\right| \leq x_{i}\left(\tau_{1}\right)$ established, which

$$
\varphi_{\alpha}\left(x_{1}, x_{2}\right)=x_{1}+\frac{1}{2-\alpha} \operatorname{sgn}\left(x_{2}\right)\left|x_{2}\right|^{2-\alpha}
$$

The smaller $\mathcal{E}$ is, the shorter the convergence time will be, the tracking smaller error will be. However, ${ }^{\varepsilon}$ is too small, so that the derivative of the differentiator has a large peak near the initial time. ${ }^{\varepsilon}$ worth selecting is detailed in the text. [13]

For this type of differentiator, in addition to obtaining the derivatives of the general continuous derivative signals, since the convergence time and the tracking error is sufficiently small, by selecting $\varepsilon \in\left(0, \varepsilon^{*}\right)$ the derivative of some piecewise continuous signal can also be obtained.

\section{$5 \quad$ Simulation Analysis}

The Second Order differentiator in finite time is designed as follows : $\left\{\begin{array}{l}\dot{x}_{1}=x_{2} \\ \varepsilon^{2} \dot{x}_{2}=-\operatorname{sat}\left\{\left(x_{1}-v(t)+\frac{3}{5}\left(\varepsilon x_{2}\right)^{5 / 3}\right)^{1 / 5}\right\}-\operatorname{sat}\left\{\left(\varepsilon x_{2}\right)^{1 / 3}\right\} \\ \operatorname{sat}(x)=\left\{\begin{array}{cc}x, & |x|<1 \\ \operatorname{sgn}(x), & |x| \geq 1\end{array}\right.\end{array}\right.$

Among them, $\varepsilon=0.004 ; x_{1}$ is the tracking of the signal, ${ }^{x_{2}}$ an estimate of the first derivative of the signal; Input signal $v(t)=10 \sin t$; The initial value of the 
differentiator are $x_{1}(0)=0, x_{2}(0)=0$. The simulation results are shown in the figure 2 .

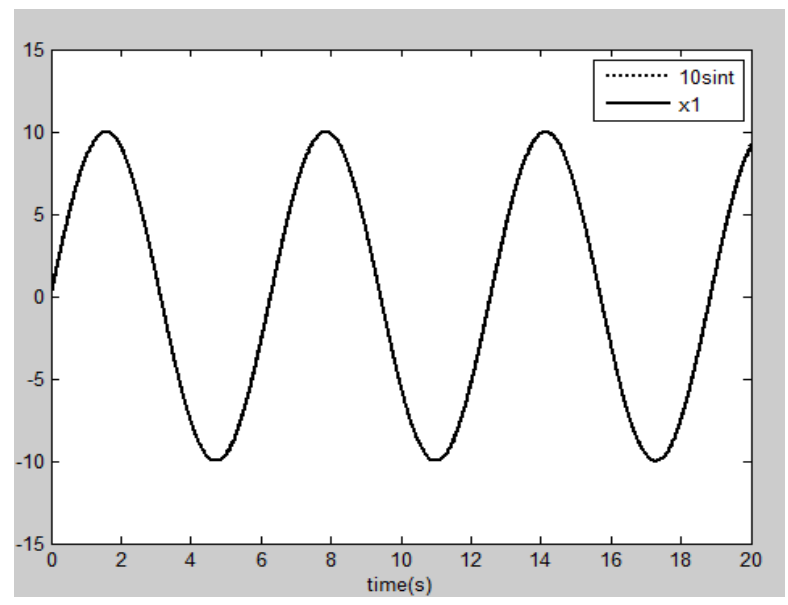

Fig. 2. Finite time convergence differentiator signal tracking

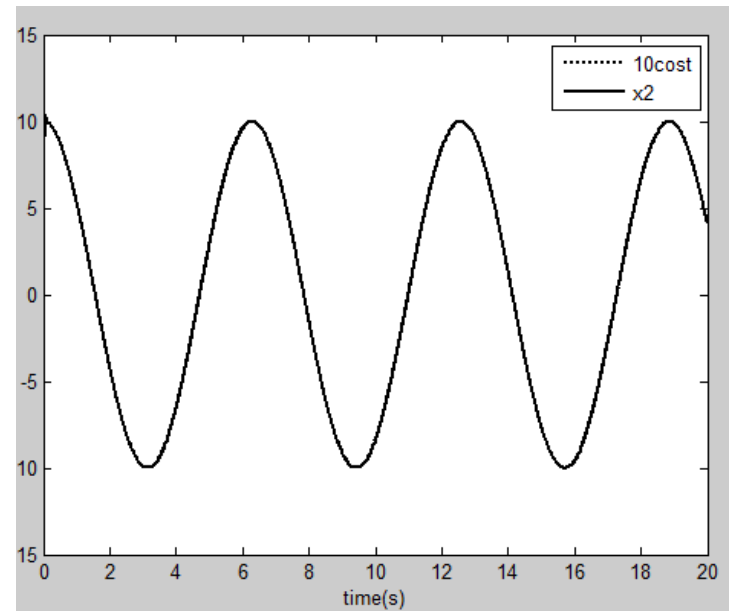

Fig. 3. Finite-time convergence differentiator derivative estimation

The differentiator realizes filtering and derivation of the signal, and has a fast convergence speed. For rigid double-joint robotic arm in planar motion, if ignoring gravity, the dynamic equation is 


$$
\left[\begin{array}{ll}
M_{11} & M_{12} \\
M_{21} & M_{22}
\end{array}\right]\left[\begin{array}{l}
\ddot{q}_{1} \\
\ddot{q}_{2}
\end{array}\right]+\left[\begin{array}{cc}
-h \dot{q}_{2} & -h\left(\dot{q}_{1}+\dot{q}_{2}\right) \\
h \dot{q}_{1} & 0
\end{array}\right]\left[\begin{array}{l}
\dot{q}_{1} \\
\dot{q}_{2}
\end{array}\right]+\left[\begin{array}{l}
N_{1} \\
N_{2}
\end{array}\right]=\left[\begin{array}{l}
u_{1} \\
u_{2}
\end{array}\right]
$$

It can be converted to

$$
\left[\begin{array}{l}
\ddot{q}_{1} \\
\ddot{q}_{2}
\end{array}\right]=\left[\begin{array}{ll}
M_{11} & M_{12} \\
M_{21} & M_{22}
\end{array}\right]^{-1}\left\{\left[\begin{array}{l}
u_{1} \\
u_{2}
\end{array}\right]-\left[\begin{array}{c}
N_{1} \\
N_{2}
\end{array}\right]\left[\begin{array}{cc}
-h \dot{q}_{2} & -h\left(\dot{q}_{1}+\dot{q}_{2}\right) \\
h \dot{q}_{1} & 0
\end{array}\right]\left[\begin{array}{l}
\dot{q}_{1} \\
\dot{q}_{2}
\end{array}\right]\right\}
$$

where

$$
\begin{aligned}
& M_{11}=a_{1}+2 a_{3} \cos q_{2}+2 a_{4} \sin q_{2}, M_{22}=a_{2} \\
& M_{21}=M_{12}=a_{2}+a_{3} \cos q_{2}+a_{4} \sin q_{2} \\
& N_{1}=\left(m_{1}+m_{e}\right) l_{1} \cos q_{2}+m_{e} l_{c 1} \cos \left(q_{1}+q_{2}\right) \\
& N_{2}=m_{e} l_{c 1} \cos \left(q_{1}+q_{2}\right) \\
& h=a_{3} \sin q_{2}-a_{4} \cos q_{2} \\
& a_{1}=I_{1}+m_{1} l_{c 1}^{2}+I_{e}+m_{e} l_{c e}^{2}+m_{e} l_{1}^{2} a_{2}=I_{e}+m_{e} l_{c e}^{2} \\
& a_{3}=m_{e} l_{1} l_{c e} \cos \delta_{e}, a_{4}=m_{e} l_{1} l_{c e} \sin \delta_{e}
\end{aligned}
$$

Let

$$
m_{1}=1, m_{e}=2, l_{1}=1, l_{c 1}=0.5, l_{c e}=0.6, I_{1}=0.12, I_{e}=0.25, \delta_{e}=\frac{\pi}{6}
$$

Among them $m_{1}$ is the quality of the first section of the arm, $m_{e}$ is the quality of the second section of the arm $l_{1}$ is the length of the first section of the arm, $l_{c 1}$ is the 
length of the second section of the arm. $I_{c e}$ is distance from the center of mass to the second joint, $I_{1}$ is the moment of inertia of the first arm, $I_{e}$ is the moment of inertia of the second arm, $\mathcal{\delta}_{e}$ is the angle between the center of mass and the second arm.

$$
y=\left[\begin{array}{ll}
q_{1} & q_{2}
\end{array}\right]^{T}, u=\left[\begin{array}{ll}
u_{1} & u_{2}
\end{array}\right]^{T}, x=\left[\begin{array}{llll}
q_{1} & \dot{q}_{1} & q_{2} & \dot{q}_{2}
\end{array}\right]
$$

The goal of controlling is to make the output of the system ${ }^{q_{1}}, q_{2}$ track the desired trajectory $y_{d 1}=\sin t, y_{d 2}=\sin t$.

Define membership function as

$$
\begin{aligned}
& \mu_{F_{i}^{1}}\left(x_{i}\right)=\exp \left(-\frac{1}{2}\left(\frac{x_{i}+1.25}{0.6}\right)^{2}\right), \mu_{F_{i}^{2}}\left(x_{i}\right)=\exp \left(-\frac{1}{2}\left(\frac{x_{i}}{0.6}\right)^{2}\right) \\
& \mu_{F_{i}^{3}}\left(x_{i}\right)=\exp \left(-\frac{1}{2}\left(\frac{x_{i}-1.25}{0.6}\right)^{2}\right), i=1,2,3,4
\end{aligned}
$$

Let

$$
\begin{aligned}
& \lambda_{1}=30, \lambda_{2}=30, K_{0}=5 I_{2},{ }_{0}=0.1, \eta_{f_{i}}=0.5, \eta_{g_{i j}}=0.5, \eta_{0}=0.001, \\
& \delta(0)=0, \bar{\varepsilon}_{g}=\left[\begin{array}{ll}
0.2 & 0.2 \\
0.2 & 0.2
\end{array}\right], \bar{\varepsilon}_{f}=\left[\begin{array}{ll}
0.2 & 0.2
\end{array}\right]^{T} .
\end{aligned}
$$

The oscillation link can be written in its state-space form as

$$
\left\{\begin{array}{l}
\dot{x}_{1}=x_{2} \\
\dot{x}_{2}=-\omega_{n}^{2}\left(x_{1}-v(t)\right)-2 \zeta \omega_{n} x_{2}
\end{array}\right.
$$

Let $\omega_{n}=1000, \zeta=0.7, v(t)$ be the position signal for the robotic arm, ${ }^{x_{2}}$ is the estimation of the first derivative of it.

The Second Order differentiator in finite time is designed as follows, 


$$
\text { Let } \varepsilon=0.0001 v(t) \text { be the position signal for the robotic arm, }{ }^{x_{2}} \text { is the estimation }
$$
of the first derivative of it.
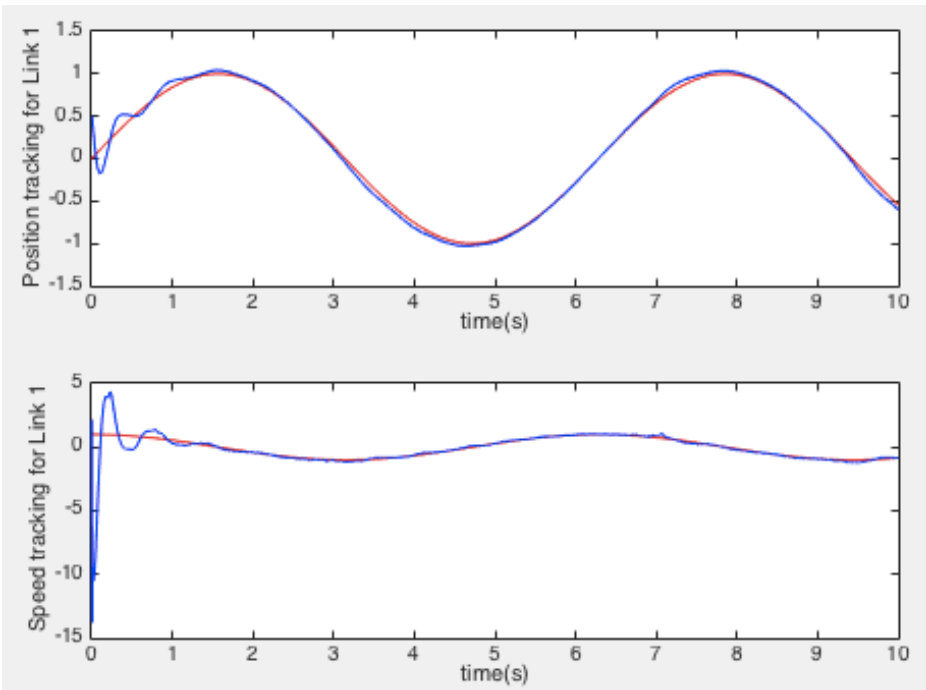

Fig. 4. Tracking curves of position and velocity with the oscillation for link 1
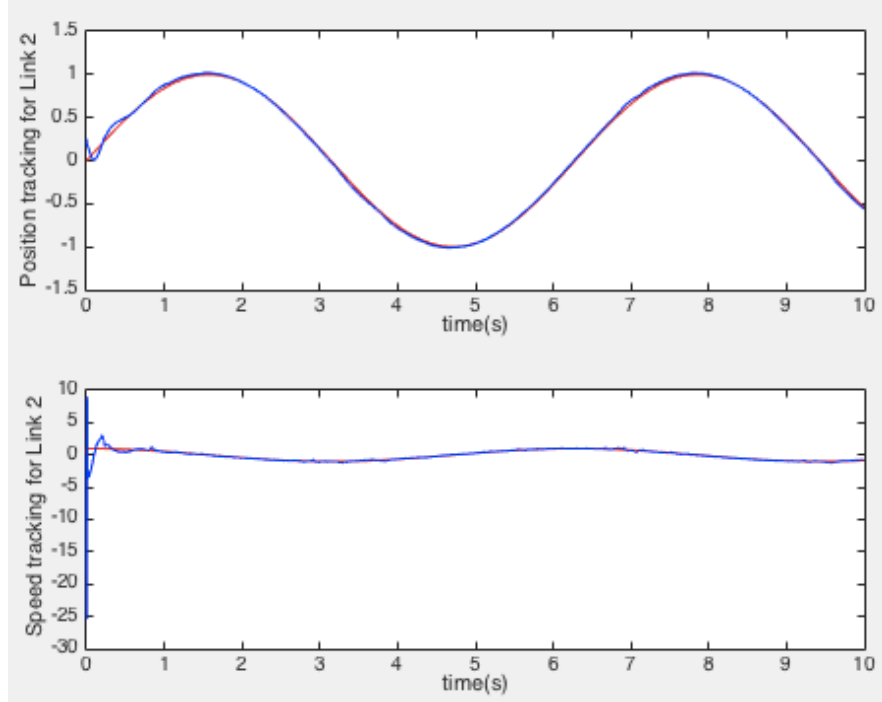

Fig. 5. Tracking curves of position and velocity with the oscillation for link 2 

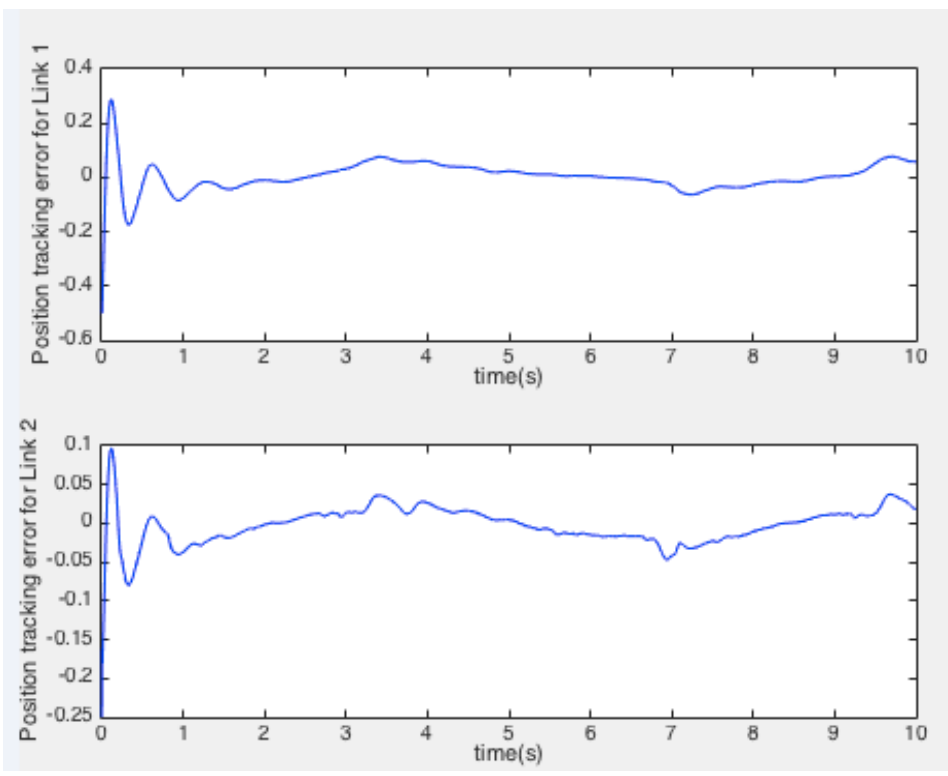

Fig. 6. Error of input signal and tracking signal based on oscillator

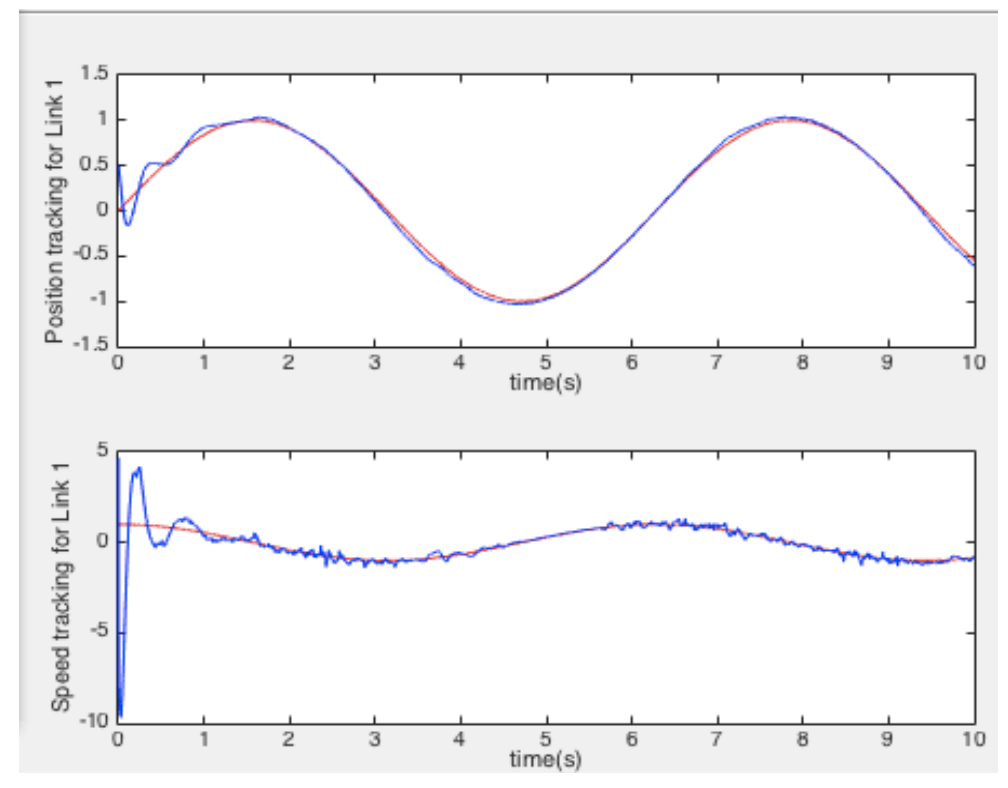

Fig. 7. Tracking curves of position and velocity with the differential for link 1 

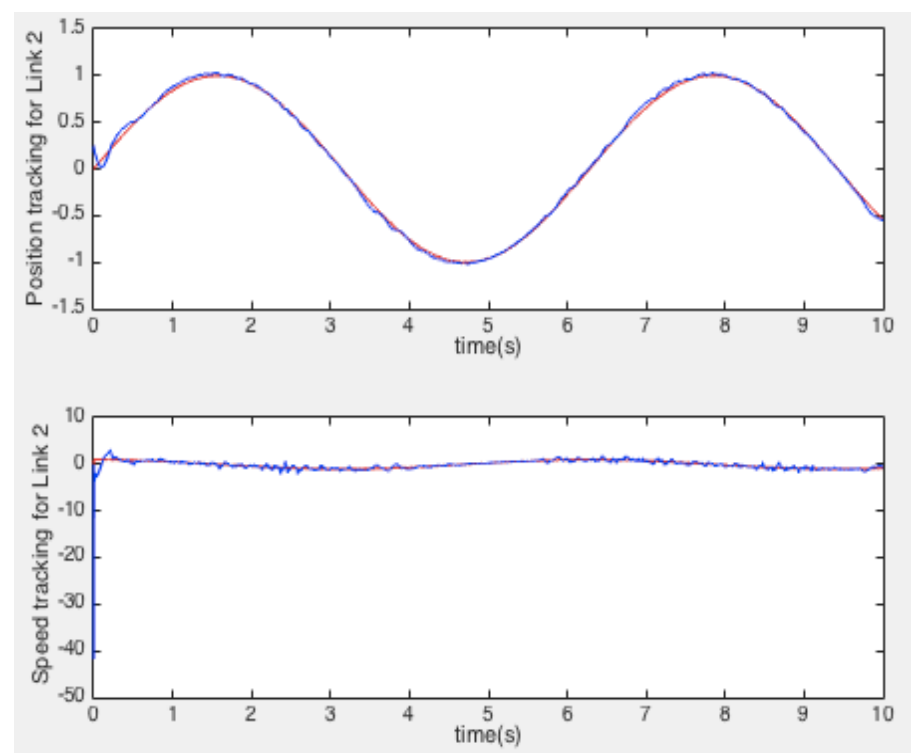

Fig. 8. Tracking curves of position and velocity with the differential for link 2
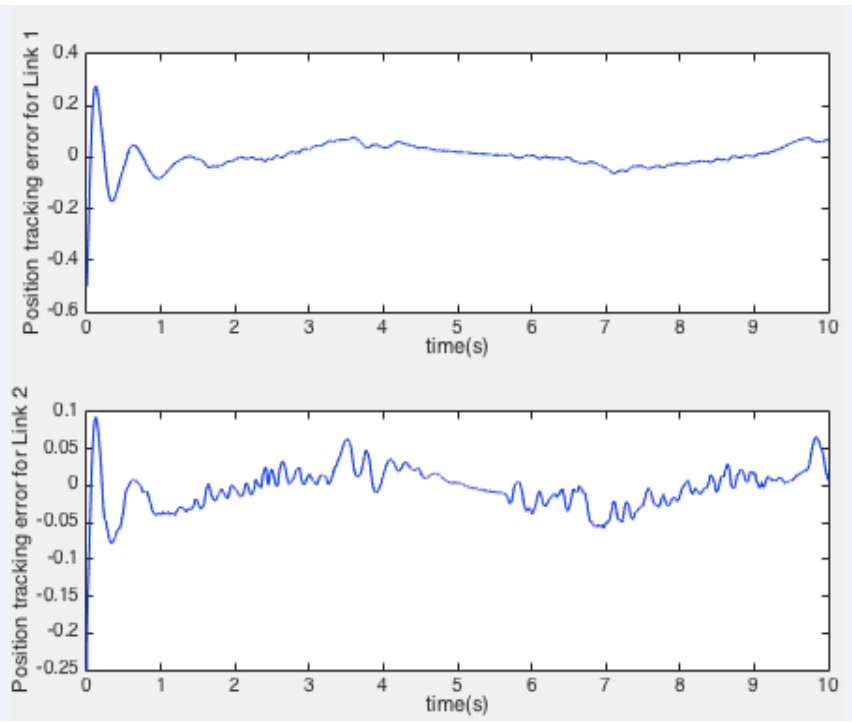

Fig. 9. Error of input signal and tracking signal based on differentiator

As shown in the simulation results, the adopting of the second-order oscillator and differentiator that converges in finite time in adaptive fuzzy controller are both able to track the trajectory smoothly, and the error is relatively small. Although there existing 
fluctuations in the early stage of position and speed tracking for link 1 and link 2, in the later period it can track the given trajectory smoothly.

\section{Conclusion}

Because fuzzy control system does not have to depend on accurate mathematical model, it is suitable for solving the control problem of the robotic arm. The fuzzy control system requires the value of the system state variables, though certain system state variables cannot or hardly be measured directly, hence introducing the second-order oscillator and the Second Order differentiator in finite time to obtain state variables.

In this paper, by using the designed adaptive fuzzy controller, the trajectory tracking control for robotic arm with high accuracy was realized. The simulation results show that the designed adaptive fuzzy controller for tracking robotic arm possessed high flexibility and adaptability. It is easy to realize, simple in structure, and also has a good value for the robotic application.

\section{$7 \quad$ Acknowledgement}

This project is supported by National Natural Science Foundation of China (grant no 51775463)

\section{$8 \quad$ References}

[1] Wang Tianmiao, Tao Yong. Research status and industrialization development strategy of Chinese industrial robot[J]. Journal of Mechanical Engineering, 2014,50(9):1-13. https://doi.org/10.3901/JME.2014.09.001

[2] Ren Guohua. Mobile robot trajectory tracking and motion control [J]. Machinery Design and Manifacture, 2014, (3):100-102.

[3] Xu Z H, Lu T S. Adaptive fuzzy control for trajectory tracking of hopping robot[J]. Journal of System Simulation, 2008, 20(23):6455-6457.

[4] Khalate A A, Leena G, Ray G. An Adaptive Fuzzy Controller for Trajectory Tracking of Robot Manipulator [J]. Intelligent Control \& Automation, 2015, 02(4):364-370. https://doi.org/10.4236/ica.2011.24041

[5] Liu Guorong, et al. Direct adaptive fuzzy robust control for a class of nonlinear MIMO system[J]. Control Theory and Application,2003,20(5):697-698.

[6] Wu T S, Karkoub M, Chen H S, et al. Robust tracking observer-based adaptive fuzzy control design for uncertain nonlinear MIMO systems with time delayed states[J]. Information Sciences, 2015, 290:86-105. https://doi.org/10.1016/i.ins.2014.08.001

Wu T S, Karkoub M, Weng C C, et al. Trajectory tracking for uncertainty time delayed-state self-balancing train vehicles using observer-based adaptive fuzzy control[J]. Information Sciences, 2015, 324(C):1-22. https://doi.org/10.1016/j.ins.2015.06.019 
[7] Li Y X, Yang G H. Observer-Based Fuzzy Adaptive Event-Triggered Control Co-Design for a Class of Uncertain Nonlinear Systems [J]. IEEE Transactions on Fuzzy Systems, 2017, PP (99):1-1.

[8] Gao Y, Tong S, Li Y. Observer-based adaptive fuzzy output constrained control for MIMO nonlinear systems with unknown control directions [M]. Elsevier North-Holland, Inc. 2016.

[9] Li Y, Tong S, Li T. Observer-Based Adaptive Fuzzy Tracking Control of MIMO Stochastic Nonlinear Systems with Unknown Control Directions and Unknown Dead Zones[J]. IEEE Transactions on Fuzzy Systems, 2015, 23(4):1228-1241. https://doi.org/10.1109/TFUZZ.2014.2348017

[10] Wan Min, An Lingzhi. Control system simulation research for double joints manipulator based on MATLAB [J]. Journal of Plasticity Engineering, 2017,24(6):136-142.

[11] Liu Jinkun. Robot control system and MATLAB simulation[M]. Beijing: Tsinghua University Press, 2013.

[12] Wang Xinhua, Liujinkun. Differentiator design and application-signal filtering and differentiation[M]. Publishing House of Electronics Industry,2010.

\section{Authors}

Min Wan received the B.S. degree in Automation from Dalian Maritime University, Dalian, China, in 2000 and the M.S. degree in Detection Technology and Automation Device from University of Electronic Science and Technology, Chengdu, China, in 2006. She is currently working toward the Ph.D. degree with the Institute of Mechanical and Electrical Engineering, Southwest Petroleum University, Chengdu, China. Her current research interests include fuzzy logic system, stochastic adaptive control, and robot control.

Qinglan Tian received the B.S. degree in Automation from Shanghai University Of Engineering Science, Shanghai, China, in 2016. She is currently working toward the M.S. degree with the Instrumentation Engineering, Southwest Petroleum University, Sichuan, China. Her current research interest includes fuzzy control and adaptive control.

Chuanhong Sun is currently working toward the B.S. degree with the Process Equipment and Control Engineering, Southwest Petroleum University, Sichuan, China. He current research interest includes process control and robot control.

Xiuyuan Yi is currently working toward the B.S. degree with the Process Equipment and Control Engineering, Southwest Petroleum University, Sichuan, China. He current research interest includes process control and robot control.

Article submitted 2018-06-19. Resubmitted 2019-01-22. Final acceptance 2019-01-23. Final version published as submitted by the authors. 